\title{
High PD-L1 expression was associated with poor prognosis in 870 Chinese patients with breast cancer
}

\author{
Tao Qin ${ }^{1, *}{ }^{,}$Yin-duo Zeng ${ }^{1,2, *}$, Ge Qin ${ }^{1, *}$, Fei Xu ${ }^{1, *}$, Jia-bin Lu ${ }^{1}$, Wen-feng Fang ${ }^{1}$, Cong \\ $X_{\text {Xue }}{ }^{1}$, Jian-hua Zhan ${ }^{1}$, Xin-ke Zhang ${ }^{1}$, Qiu-fan Zheng ${ }^{1}$, Rou-jun Peng ${ }^{1}$, Zhong-yu \\ Yuan ${ }^{1}$, Li Zhang ${ }^{1}$ and Shu-sen Wang ${ }^{1}$ \\ ${ }^{1}$ Sun Yat-sen University Cancer Center, The State Key Laboratory of Oncology in South China, Collaborative Innovation \\ Center for Cancer Medicine, Guangzhou, Guangdong, P. R. China \\ ${ }^{2}$ Sun Yat-sen University Sun Yat-sen Memorial Hospital, Guangzhou, Guangdong, P. R. China \\ * These authors have contributed equally to this work \\ Correspondence to: Shu-sen Wang, email: wangshs@sysucc.org.cn
}

Li Zhang, email: zhanglib@mail.sysu.edu.cn

Zhong-yu Yuan, email: yuanzhy@sysucc.org.cn

Keywords: PD-L1, breast cancer, ER, PR, prognosis, nomogram

Received: July 07, $2015 \quad$ Accepted: August 22, 2015

Published: September 10, 2015

This is an open-access article distributed under the terms of the Creative Commons Attribution License, which permits unrestricted use, distribution, and reproduction in any medium, provided the original author and source are credited.

\section{ABSTRACT}

Background: To investigate the role of PD-L1 expression in tumor recurrence and metastasis in Chinese patients with breast cancer.

Methods: Suitable tissue samples were available from 870 patients with breast cancer. Paraffin-embedded tumor sections were stained with PD-L1 antibody. The correlations between PD-L1 expression and clinical characteristics, ER/PR/HER2 status and survival parameters were analyzed. Kaplan-Meier and univariate Cox proportional hazards model analyses were used to compare the survival of patients with high PD-L1 expression and patients with no PD-L1 expression.

Results: The median follow-up time was 98 months(range, 17-265 months).The positive rate of PD-L1 expression in breast cancer was $21.7 \%(189 / 870)$. PD-L1 high expression was inversely associated with larger tumor size, higher tumor grade, more positive lymph node number, as well as negative ER and PR status. PD-L1 expression was particularly higher in TNBC compared with non-TNBC, although no statistical significance was observed. Nomogram logistic regression results based on clinical and pathological features showed that the following factors were more likely associated with high PD-L1 expression: patient age younger than $\mathbf{3 5}$ years, larger tumor size, lymphovascular invasion and advanced stage. Our data indicated that patients with high PD-L1 expression had poor DFS, DMFS and overall survival compared with those with no PD-L1 expression. Univariate Cox proportional hazards model analysis showed that PD-L1 was an independent prognostic factor for tumor prognosis.

Conclusions: PD-L1 expression is an important indicator of unfavorable prognosis in breast cancer patients.

\section{INTRODUCTION}

Breast cancer is currently the second leading cause of tumor-related death for females worldwide [1]. Despite the development of treatments for breast cancer, more than $50 \%$ of invasive breast cancer patients have developed distant metastases within ten years, causing treatment failure [2]. Interestingly, in recent years, immune therapy has become an emerging effective treatment for several cancers.

Recently, extended adjuvant endocrine therapy was shown to benefit patients with hormone receptor-positive breast cancer in the large sample size randomized ATLAS study [3]. However, the recurrence of breast cancer 
remained high in realistic clinical practice. Therefore, finding an effective biomarker to select patients with breast cancer who are at a high risk for tumor recurrence or metastasis is an urgent task.

The risk of tumor recurrence is reasonable within the 5-yearfollow-up period following treatment with adjuvant tamoxifen; however, a number of patients with luminal B/HER2 negative subtype tumors presented tumor recurrence after the 5-year period following treatment with tamoxifen [2]. Several predicting tools including Breast Cancer Index (BCI), Oncotype DX recurrence score, IHC4 [4], andHOXB13/IL17BR (H/I) [5]were used to predict the risk of late disease recurrence. Although several predicting markers have been developed, no precise factor existed that could predict the long-term survival of breast cancer patients. The primary cause of recurrence or metastases from operable breast cancer was immune resistance produced by the tumor. Immune activity plays a role in cancer metastatic immune checkpoint agonist drugs have been approved for use in treating melanoma. Previously, the PD1/PD-L1 pathway was reported as the primary factor promoting tumor recurrence or metastasis. PD-L1, which is major ligand of PD-1, is expressed in a variety of cancers. Studies have shown PD-L1 inhibition is effective treating in many malignant tumors such as non-small cell lung cancer (NSCLC) [6], renal cancer [7], triple-negative breast cancer [8] and bladder cancer [9, 10].

Tumors can cause changes in the microenvironment and may evoke an imbalance of immunomodulation between tumor growth and host surveillance, finally promoting tumor metastasis. PD-1/PD-L1 checkpoint antibodies help to reconstruct the balance between the host and tumor, resulting in dynamic and durable tumor regression. Nivolumab and pembrolizumab are two anti-PD-1 antibodies that are currently approved for use in clinical treatments for melanoma. MPDL3280A, MEDI4736, and BMS-936559 were investigated for potential treatment of other tumor types such as metastatic melanoma, NSCLC, and breast cancer [11]. Currently, the PD1/PD-L1 pathway in breast cancer has been well studied [12, 13]. However, the molecular regulatory mechanism of PD-L1 in different subtypes of breast cancer remains unknown [12]. As previous studies have indicated, the positive rate of intra tumor PD-L1 expression ranged from $20 \%$ to $60 \%$ of breast cancer patients [14, 15]. A large randomized study showed that PD-L1 blockade prolonged survival in triple-negative breast cancer that lacked an effective treatment [16]. However, PD-L1 expression reflected inconsistent survival outcomes in breast cancer. Two studies showed that tumors with a high level of PD-L1mRNA expression correlated with significantly better recurrence-free survival in breast cancer patients $[17,18]$, whereas other studies showed that high PD-L1 expression was significantly associated with poorer survival [14]. Recently, a genomic analysis of the PD-L1 gene in breast cancer showed that the PDL1 gene was inversely associated with the ESR1 gene in 5,454 breast cancers profiled using DNA microarrays [18]. IHC studies strongly suggested that PD-L1 expression was an unfavorable factor that was associated with decreased disease-free survival and overall survival $[14,15]$. In addition, several studies showed that PD-L1 was more highly expressed in triple-negative breast cancer and HER2-positive breast cancer [19]. Therefore, studying PD-L1 expression in Eastern Asian patients with breast cancer is of significance. This study aimed to explore the role of PD-L1 expression in the prognosis of 870 Eastern Asian breast cancer patients.

\section{RESULTS}

\section{Patient characteristics}

In total, 870 Eastern Asian patients with invasive breast cancer were enrolled in this study. The median age at diagnosis was 47.0 years (range, 21-84 years). The baseline characteristics of these patients are listed in Table 1. PD-L1 expression was more common in patients with tumors that were larger than $2 \mathrm{~cm}$, with lymphvascular invasion, a higher tumor grade, as well as negative ER and PR status.

\section{PD-L1 expression and patient baseline clinical characteristics}

PD-L1 was found at the membrane or in the cytoplasm (or both) of tumor cells by immunohistochemical staining (Figure 1). IntratumorPD-L1 expression was observed in 189 (21.7\%) patients. The relation of PD-L1 expression with various clinicopathological parameters is shown in Table 1.

In addition, patient characteristics were more associated with the probability of positive PD-L1 expression. Therefore, we used a nomogram to predict which subtype of patients was more likely to present high PD-L1 expression. Nomogram analysis focused on clinical characteristics to predict PD-L1 positive. The results was to distinguish which subtype of patients with breast cancer should be detect PD-L1 concurrent with ER/ PR/HER2 after breast surgery. in addition, our data also showed that patients with the following characteristics were more likely associated with high PD-L1 expression: larger tumor size, lymphovascular invasion, advanced nodal stage, negative ER status, negative PR status as well as HER2 status (Figure 2). 

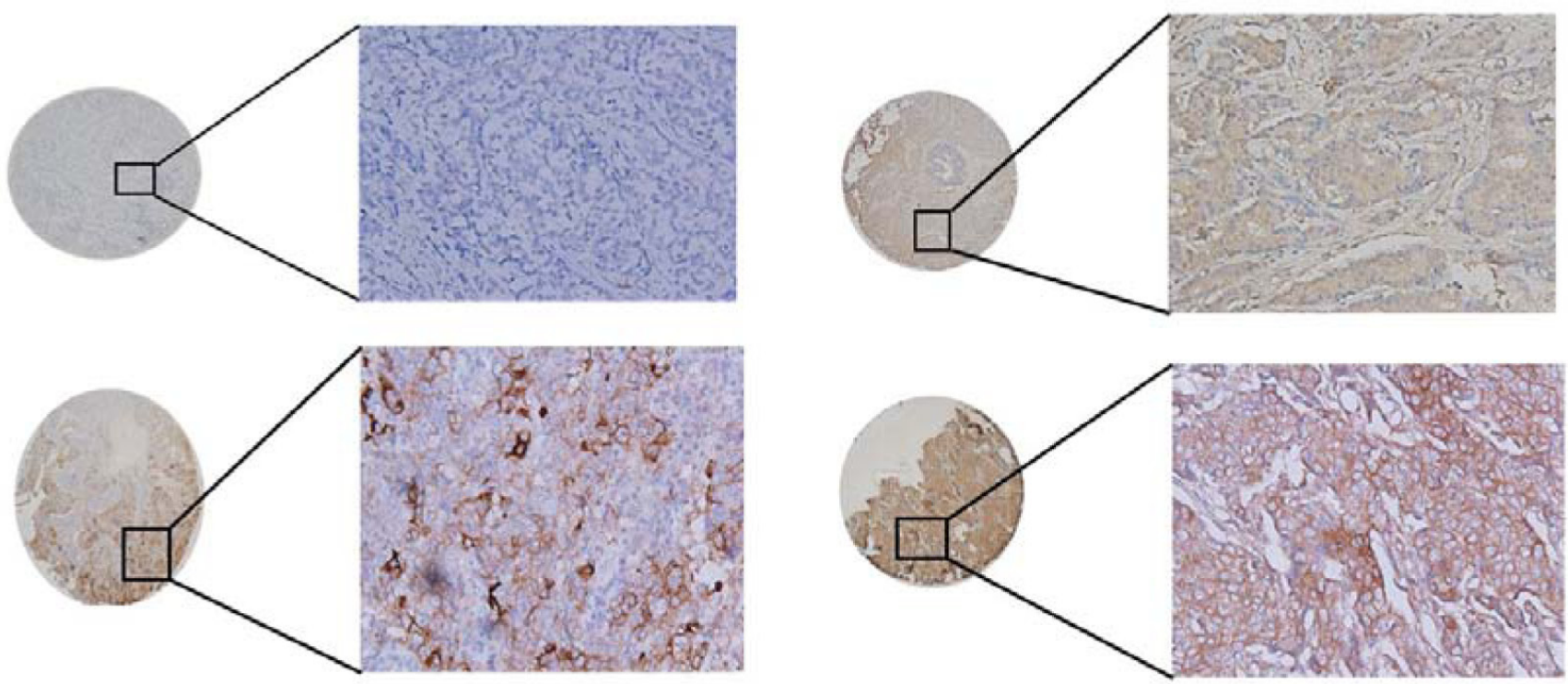

\begin{tabular}{l|l}
$A$ & $B$ \\
\hline$C$ & $D$
\end{tabular}

Figure 1: PD-L1 expression in breast cancer tissues (A, PD-L1 negative; B, C\&D, PD-L1 positive).

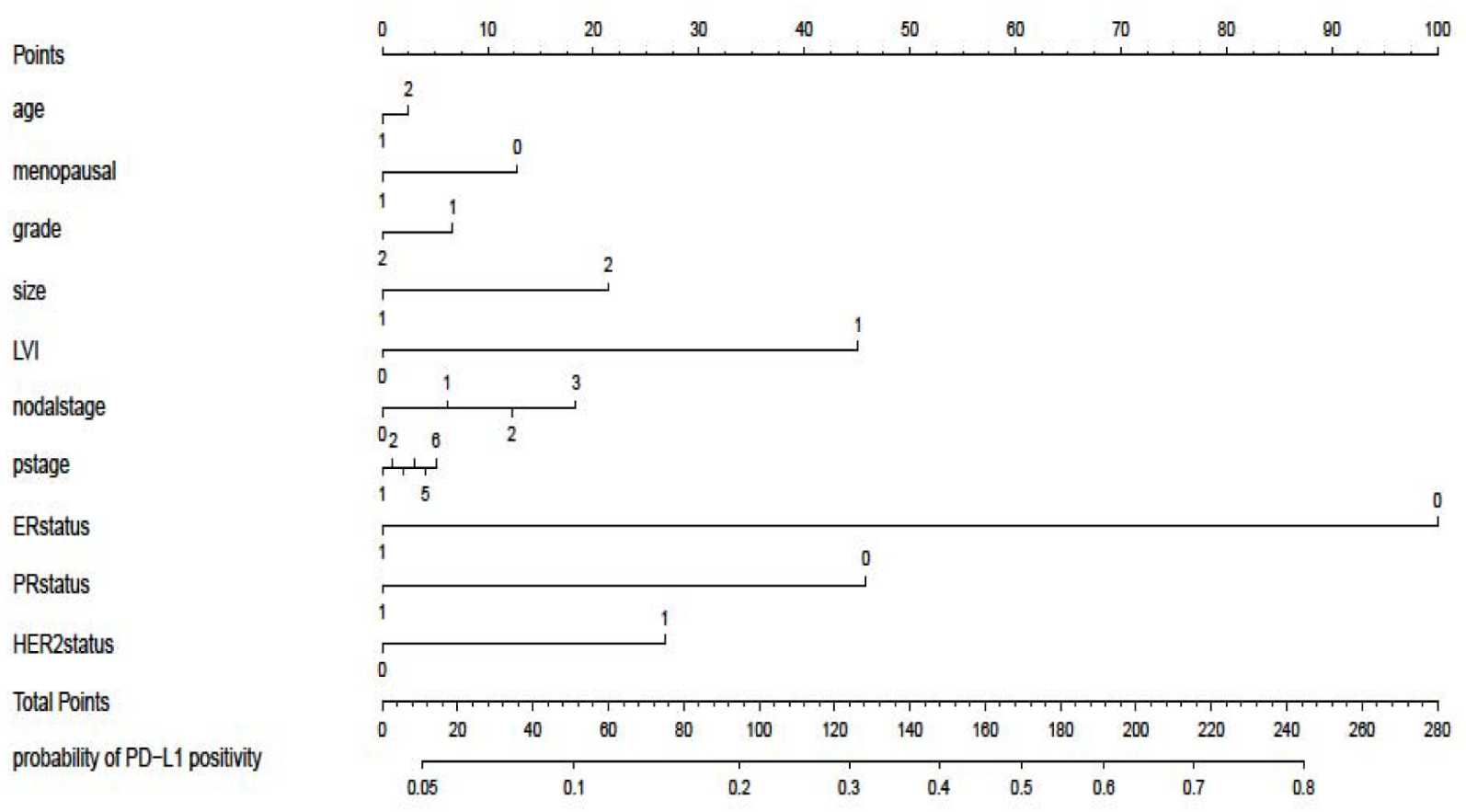

Figure 2: Nomogram predicting patients with PD-L1-positive tumors according to varied clinical characteristics. LVI, lymphovascular invasion. 
Table 1: PD-L1 expression levels of 870 breast cancer patients.

\begin{tabular}{|c|c|c|c|}
\hline \multirow[b]{2}{*}{ Variable } & \multicolumn{2}{|c|}{$P D-L 1$ expression $[n(\%)]$} & \multirow{2}{*}{$\begin{array}{c}P- \\
\text { value }^{a}\end{array}$} \\
\hline & negative & positive & \\
\hline Medianage (years) & \multicolumn{2}{|c|}{$47(21-84)$} & \\
\hline Tumor size $(\mathrm{mm})$ & & & 0.002 \\
\hline$\leq 20$ & $238(84.4)$ & $44(15.6)$ & \\
\hline$>20$ & $38(66.7)$ & $19(33.3)$ & \\
\hline Stage & & & 0.257 \\
\hline I & $129(83.2)$ & $26(16.8)$ & \\
\hline II & $461(77.2)$ & $136(22.8)$ & \\
\hline III & $91(77.1)$ & $27(22.9)$ & \\
\hline Histologicalgrade $\mathrm{a}^{\mathrm{a}}$ & & & 0.013 \\
\hline 1 & $32(86.5)$ & $5(13.5)$ & \\
\hline 2 & $279(82.5)$ & $59(17.5)$ & \\
\hline 3 & $370(74.7)$ & $125(25.3)$ & \\
\hline Positive lymph nodes & & & 0.533 \\
\hline 0 & $334(78.2)$ & $93(21.8)$ & \\
\hline $1-3$ & 190(80.9) & $45(19.1)$ & \\
\hline $4-9$ & $93(76.9)$ & $28(23.1)$ & \\
\hline$\geq 10$ & $64(73.6)$ & $23(26.4)$ & \\
\hline Lymph node ratio & & & 0.470 \\
\hline$<0.20$ & $169(80.1)$ & $42(19.9)$ & \\
\hline $0.21 \leq \mathrm{xx}<0.65$ & $120(78.4)$ & $33(21.6)$ & \\
\hline$>0.65$ & $58(73.4)$ & $21(26.6)$ & \\
\hline Lymphovascular invasion & & & 0.015 \\
\hline No & $664(78.8)$ & $179(21.2)$ & \\
\hline Yes & $16(59.3)$ & $11(40.7)$ & \\
\hline Receptor status & & & \\
\hline Estrogen & & & $<0.001$ \\
\hline Positive & $570(90.5)$ & $60(9.5)$ & \\
\hline Negative & $111(46.30)$ & $129(53.8)$ & \\
\hline Progesterone & & & $<0.001$ \\
\hline Positive & $555(90.0)$ & $62(10.0)$ & \\
\hline Negative & $126(49.8)$ & $127(50.2)$ & \\
\hline HER2 & & & 0.529 \\
\hline Negative & $668(78.4)$ & $184(21.6)$ & \\
\hline Positive & $13(72.2)$ & $5(27.8)$ & \\
\hline Ki67 index ${ }^{b}$ & & & 0.028 \\
\hline$\leq 14 \%$ & $86(69.9)$ & $37(30.1)$ & \\
\hline$>14 \%$ & $349(79.3)$ & $91(20.7)$ & \\
\hline Neo-adjuvant chemotherapy & & & 0.428 \\
\hline
\end{tabular}




\begin{tabular}{lccc}
\hline No & $625(79.6)$ & $170(21.4)$ & \\
Yes & $56(74.7)$ & $19(25.3)$ & \\
Chemotherapy & & & 0.109 \\
No & $78(84.8)$ & $14(15.2)$ & \\
Yes & $603(77.5)$ & $175(22.5)$ & \\
Radiotherapy & & & 0.797 \\
Yes & $521(77.8)$ & $14(22.2)$ & \\
No & $23(74.2)$ & $8(25.8)$ & \\
Endocrinetherapy & & & $<\mathbf{0 . 0 0 1}$ \\
No & $192(61.9)$ & $118(38.1)$ & \\
Yes & $489(87.3)$ & $71(12.7)$ & \\
Subtypes & & & $<\mathbf{0 . 0 0 1}$ \\
Luminal A & $284(88.5)$ & $37(11.5)$ & \\
Luminal B/HER2 negative & $287(91.4)$ & $27(8.6)$ & \\
Luminal B/ HER2 positive & $12(92.3)$ & $1(7.7)$ & \\
Triple-negative & $98(44.1)$ & $124(55.9)$ & \\
\hline
\end{tabular}

a, HER2, human epidermal growth factor receptor 2;b, 563 patients with known ki67expression.

\section{Relation between PD-L1 expression and prognosis}

The median follow-up time was 98 months(range,

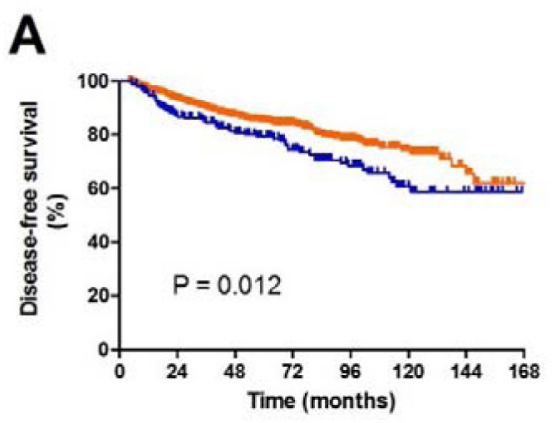

C

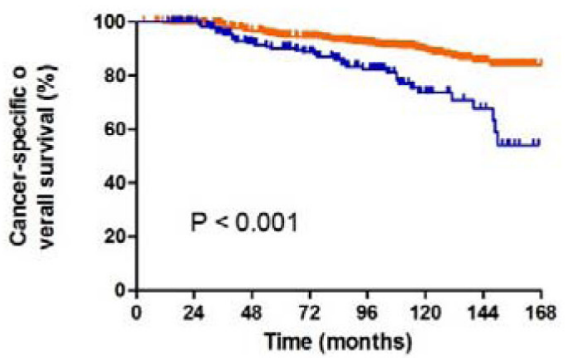

17-265 months). PD-L1-positive breast cancer patients had significantly shorter DMFS, DFS and OS values than those of PD-L1-negative patients (Figure. 3). The 5-year DMFS for PD-L1-positive patients was significantly poorer than

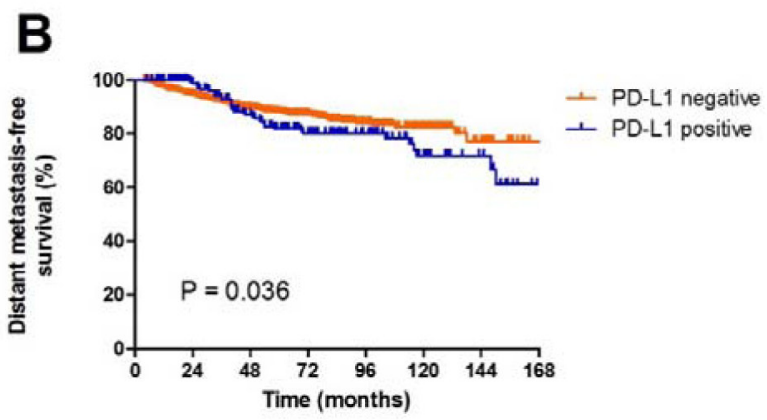

D

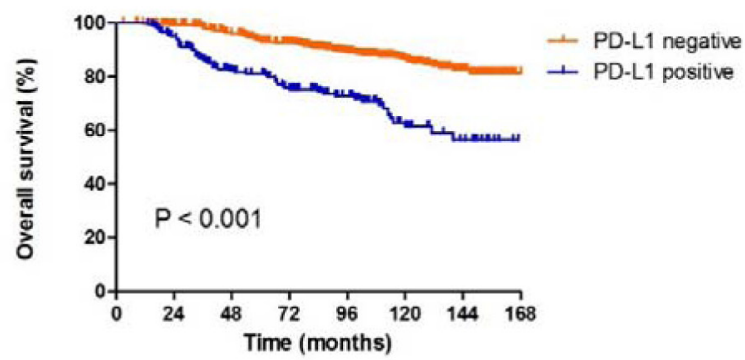

Figure 3: Survival analysis according PD-L1 expression(A, DFS; B, DMFS; C, cancer-specific OS; D, OS) 
Table 2: Univariate and multivariate analyses for DFS.

\begin{tabular}{|c|c|c|c|c|c|c|c|c|}
\hline \multirow{3}{*}{ Variables } & \multicolumn{4}{|c|}{ Univariate } & \multicolumn{4}{|c|}{ Multivariate } \\
\hline & \multirow[b]{2}{*}{$H R$} & \multicolumn{2}{|c|}{$95 \% C I$} & \multirow[b]{2}{*}{ P-value } & \multirow[b]{2}{*}{$H R$} & \multicolumn{2}{|c|}{$95 \% C I$} & \multirow[b]{2}{*}{ P-value } \\
\hline & & Lower & Upper & & & Lower & Upper & \\
\hline Age (continue) & 0.991 & 0.977 & 1.005 & 0.203 & & & & \\
\hline $\begin{array}{l}\text { Tumor size } \\
\text { (>20 vs. } \leq 20 \mathrm{~mm})\end{array}$ & 2.530 & 1.688 & 3.791 & $<0.001$ & 1.960 & 1.302 & 2.951 & 0.001 \\
\hline Grade(III vs. I-II) & 1.132 & 0.861 & 1.488 & 0.373 & & & & \\
\hline $\begin{array}{l}\text { LN status } \\
\text { (pos vs. neg) }\end{array}$ & 4.084 & 2.857 & 5.839 & $<0.001$ & $3, .731$ & 2.604 & 5.346 & $<0.001$ \\
\hline ERstatus (pos vs. neg) & 0.900 & 0.652 & 1.242 & 0.521 & & & & \\
\hline PR status (posvs. neg) & 0.943 & 0.685 & 1.299 & 0.721 & & & & \\
\hline $\begin{array}{l}\text { HER2 status (pos vs. } \\
\text { neg) }\end{array}$ & 1.181 & 0.971 & 1.437 & 0.097 & & & & \\
\hline ki67 (>14\% vs. $\leq 14 \%)$ & 1.283 & 0.775 & 2.214 & 0.333 & & & & \\
\hline $\begin{array}{l}\text { PD-L1 status (pos vs. } \\
\text { neg) }\end{array}$ & 1.503 & 1.091 & 2.071 & 0.013 & 1.386 & 1.003 & 1.916 & 0.048 \\
\hline TNBC (yes vs. no) & 0.987 & 0.704 & 1.384 & 0.942 & & & & \\
\hline
\end{tabular}

Abbreviations: LN, lymph node; pos, positive; neg, negative; HER2, human

epidermal growth factor receptor type 2; TNBC, triple-negative breast cancer.

those withPD-L1-negative patients( $83 \%$ vs. $88 \%, P=$ 0.036). When the patients were stratified in terms of PDL1 status, the five-year DFS values for PD-L1-positive and PD-L1-negative patients were $78.6 \%$ vs. $84.9 \%(P=$ $0.012)$, respectively. The cancer-specific overall survival for PD-L1 positive patients was significantly poorer than that of PD-L1-negative patients (88\% vs. 91.5\%, $P<$ $0.001)$.

\section{Univariate and multivariate analyses}

PD-L1 expression and patient's characteristics including age, tumor size, tumor grade, positive lymph node number, lymph node ratio, ER status, PR status, HER2status were included to perform univariate and multivariate analyses.

Statistically significant predictors of DFS within the univariate analysis are listed in Table 2. In univariate survival analyses, larger tumor size, positive lymph-node status, PD-L1 expression revealed unfavorable DFS for breast cancer patients.

In addition, a larger tumor size, positive lymphnode status, and PR-negative, triple negative and PDL1-positive expression were associated with poorer OS (Table 3). In the multivariate analysis, a tumor size larger than $2 \mathrm{~cm}$, positive lymph-node status and PD-L1positiveexpression proved to be independent negative prognostic factors for both DFS and OS (Table 3).

\section{DISCUSSION}

We investigated the prevalence and significance of PD-L1 expression in breast cancer. In this study of 870 breast cancer patients, the total positivity of PD-L1 was greater than $20 \%$. PD-L1 high expression was inversely associated with large tumor size, higher tumor grade, more positive lymph node number, higher lymph node ratio, negative ER/PR status. PD-L1 expression was particularly higher in TNBC compared with non-TNBC. As our data demonstrated, patients with positive PD-L1 expression had significantly decreased survival compared to those with no PD-L1 expression. Cox proportional hazards model analysis indicated that PD-L1 expression was a strong independent prognostic factor for patient prognosis.

Programmed death 1 (PD-1) is a co-inhibitory receptor that is expressed on the membranes of activated $\mathrm{T}$ and $\mathrm{B}$ cells [6] and that plays an important role in tumor immune escape $[7,8]$. The major ligand for PD-1 is PDL1, which is expressed in a variety of cancers [9]. Adaptive immune responses that includePD1/PD-L1 expression are associated with breast cancer relapse. PD1/PD-L1 is an important axis that plays important roles in the infiltration of various immune effectors and in the propensity to relapse with metastatic disease. Recent evidence suggests that activation of the PD-1/PD-L1 pathway represents one mechanism that allows tumors to elude the host immune system [16-18]. Previous studies have reported that PD-L1 is involved in the negative regulation of immune response 
Table 3: Univariate and multivariate analyses for OS.

\begin{tabular}{|c|c|c|c|c|c|c|c|c|}
\hline \multirow{3}{*}{ Variables } & \multicolumn{4}{|c|}{ Univariate } & \multicolumn{4}{|c|}{ Multivariate } \\
\hline & \multirow[b]{2}{*}{$H R$} & \multicolumn{2}{|c|}{$95 \% C I$} & \multirow[b]{2}{*}{$P$-value } & \multirow[b]{2}{*}{$H R$} & \multicolumn{2}{|c|}{$95 \% C I$} & \multirow[b]{2}{*}{$P$-value } \\
\hline & & Lower & Upper & & & Lower & Upper & \\
\hline Age (continue) & 1.005 & 0.990 & 1.021 & 0.477 & 1.249 & 0.685 & 2.276 & 0.469 \\
\hline $\begin{array}{l}\text { Tumor size } \\
(>20 \text { vs. } \leq 20 \mathrm{~mm})\end{array}$ & 2.648 & 1.679 & 4.174 & $<0.001$ & 1.880 & 1.183 & 2.990 & 0.008 \\
\hline Grade(III vs. I-II) & 1.242 & 0.916 & 1.683 & 0.163 & & & & \\
\hline $\begin{array}{l}\text { LN status } \\
\text { (pos vs. neg) }\end{array}$ & 4.718 & 3.080 & 7.226 & $<0.001$ & 2.222 & 1.9056 & 2.592 & $<0.001$ \\
\hline ER (pos vs. neg) & 0.871 & 0.755 & 1.005 & 0.059 & & & & \\
\hline PR(posvs. neg) & 0.797 & 0.687 & 0.926 & 0.003 & 0.908 & 0.420 & 1.963 & 0.806 \\
\hline $\begin{array}{l}\text { HER2 status (pos vs. } \\
\text { neg) }\end{array}$ & 1.118 & 0.881 & 1.419 & 0.360 & & & & \\
\hline ki67 $(>14 \%$ vs. $\leq 14 \%)$ & 0.831 & 0.487 & 1.417 & 0.497 & & & & \\
\hline PD-L1 (pos vs. neg) & 2.262 & 1.598 & 3.203 & $<0.001$ & 1.788 & 1.195 & 2.674 & 0.005 \\
\hline TNBC (yes vs. no) & 1.454 & 1.003 & 2.108 & 0.048 & 1.398 & 0.606 & 3.225 & 0.432 \\
\hline
\end{tabular}

Abbreviations: LN, lymph node; pos, positive; neg, negative; TNBC, triple-negative breast cancer.

binding to PD-1 receptor and results in cancer cells evading the host immune surveillance, finally promoting metastasis [10-12].

PD-L1 expression was evaluated as a predictor of unfavorable prognosis for many other malignant cancers such as NSCLC, melanoma, renal cancer, glioblastoma (GBM), ovarian cancer, and colon cancer. Regarding breast cancer, the reported positivity of PD-L1 expression in tumor cells varied. A recent study showed that PDL1 expression by immune cells was observed in $6 \%$ of tumors, while PD-L1 expression by tumor cells occurred in only $1.7 \%$ of a total of 3796 breast cancer patients [20]. However, in our study, we showed that PD-L1 expression in breast tumor cells occurred in $21.7 \%$ of all patients. Our results were consistent with PD-L1 expression results of previous reports. Moreover, statistical analysis found that PD-L1 was associated with many tumor characteristics of breast cancer. In addition, clinical characteristics closely correlated with PD-L1 expression. Ghebeh analyzed 44 patients and found that PD-L1 expression was significantly higher in ER-negative tumors, PR-negative tumors and higher-grade tumors [15]. However, due to the sensitivity ofdetectionforPD-L1, the rate of PD-L1 expression differed. Another study using immunohistochemical methods showed that high PD-L1 mRNA expression levels were more common in patients with the following characteristics: a larger tumor size, high proliferation, high tumor grade, and ER-negative and PR-negative status [18]. Our data were consistent with previous studies and demonstrated that positive PD-L1 expression was associated with tumor grade and with ER and PR status. Moreover, patients with tumor size and LVI had a higher proportion of positive PD-L1 expression. The results of our study were consistent with previous reports.

PD-L1 expression varied in different subtypes of breast cancer. One study showed higher positive rates of PD-L1 expression in different types of tumor cells, $20 \%$ in HER2-positive cells, $33 \%$ in luminal subtype cells and up to $59 \%$ in triple-negative breast cancer cells [19]. PD-L1 mRNA expression levels were higher in HER2positiveand in basal and HER2-enriched subtypes than in other subtypes [18]. PD-L1 expression was the highest in $\mathrm{TNBC}$, in contrast to a recent study that reported the highest frequency in HER2-positive breast cancers [12, 14]. In our study, patients with TNBC seemed to have a higher proportion of positive PD-L1 expression compared with patients with non-TNBC breast cancer; however, this result was not statistically significant. Our results showed that the percentages of PD-L1 expression in luminal A, luminal B/HER2 negative, luminal B/HER2 positive and TNBC were $11.5 \%, 8.6 \%, 7.7 \%$ and $55.9 \%$, respectively. Our data indicated that patients with TNBC had a similar rate of PD-L1 expression compared with previous reports.

In addition, several studies found that other malignant oncogenic genes regulated the expression of PD-L1; for example, the EGFR pathway induces PD-L1 expression [21-23]. One limitation of the present study is that no relation was found between the breast cancerassociated gene HER2 and PD-L1. Previous studies have shown that PD-L1protein or mRNA expression levels in breast cancer tumor samples were associated with large tumor size, high tumor grade, more positive lymph-node involvement, ER-negative status, PR-negative status, ERBB2-positive status, and high proliferation, as well as 
unfavorable molecular subtypes such as HER2-enriched breast cancer or TNBC [12, 14, 15, 17, 24]. However, a recent study showed that high PD-L1 mRNA levels were associated with better prognosis [18]. An in vitro study indicated that PD-L1 expression was shown to be higher in a basal type of breast cancer cells than in luminal type cells [13]. In this study, our analysis of breast tumor samples from 870 patients, we demonstrated that PD-L1 was more common in patients with the following clinical characteristics: larger tumor size, more positive lymph node involvement, higher historically tumor grade, higher ki67 index, more LVI and negative relation with both ER and PR.

Apart from our analysis, no studies have used a model to predict the probability of PD-L1 expression according to clinical variables. In our study, we used a logistic regression model help to determine those patients who were likely to have high PD-L1 expression. This finding has not reported previously. This model will help to select those specific patients that should be tested for PD-L1 expression and may be applicable for use in clinical practice.

In our study, which compared between patients with PD-L1positive and patients with PD-L1 negative, patients with positive PD-L1 expression had significantly poorer clinical outcomes including DFS, DMFS, OS and cancerspecific OS. Patients with positive PD-L1 expression had almost two times higher risks of tumor recurrence, metastasis and cancer-related death. Several pathways may activate thePD-L1 pathway.

Notably, we concluded that high PD-L1 expression is inversely associated with large tumor size, tumor grade, lymph node positive number, and ER and PR status. Furthermore, PD-L1 expression is an important prognostic indicator of unfavorable prognosis in breast cancer patients. Finally, a nomogram model is useful for predicting high PD-L1expression levels.

\section{MATERIALS AND METHODS}

\section{Ethical statement}

This study was approved by the Sun Yat-sen University Cancer Center review board.

\section{Patients}

All patients were diagnosed with invasive ductal breast cancer with pathological confirmation at our institution from April 2000 to April 2012. All patients underwent breast conservation therapy or mastectomy. These patients received adjuvant chemotherapy and/or radiotherapy as needed according to the routine clinical practice of our center. Patients with ER/PR-positive tumors received adjuvant endocrine therapy.

\section{Tissue samples}

All slides were cut from a pre-existing invasive ductal carcinoma maintained by the Moffitt tissue core facility (breast 2B). Patient clinical information including age, tumor size, lymph node involvement, tumor grade, and ER, PR, and HER2 status was collected. Additional data including neo-adjuvant chemotherapy, chemotherapy, radiotherapy and endocrine therapy were also collected.

\section{Immunohistochemical staining}

Slides were stained using a Ventana Discovery XT automated system (Ventana Medical Systems, Tucson, AZ) with proprietary reagents according to the manufacturer's protocol. Briefly, slides were deparaffinized on the automated system with EZ Prep solution (Ventana). A heat-induced antigen retrieval method was used withCell Conditioning 1 solution (Ventana). The concentration of rabbit primary antibody that reacts to PD-L1 (Cell Signaling Technology, Beverly, MA) was 1:100 in Dako antibody diluent; slides were incubated with this antibody overnight at $4^{\circ} \mathrm{C}$. Then, the slides were incubated with Ventana Omni Mapanti-rabbit secondary antibody for 60 min. AVentana Chromo MapKit was used for antibody detection, and then the slides were counterstained with hematoxylin. Next, the slides were dehydrated and cover slipped as per normal laboratory protocol. All slides were independently examined by two pathologists; both of whom had no prior knowledge of the clinical parameters of the patient. Discrepancies were resolved through the simultaneous re-examination of the slides using a doubleheaded microscope by both pathologists. PD-L1-positive scoring denoted staining of over $5 \%$ of the tumor cell membrane with or without cytoplasm staining.

\section{Statistical analysis}

The patient distribution and clinical features between PD-L1-positive and PD-L1-negative tumors were compared by chi-square test, Wilcoxon rank-sum test, or two-sample t-test as appropriate. The primary endpoint for this analysis was disease-free survival (DFS), which is defined as the length of time from the date of surgery on the primary tumor to local, regional, or distant recurrence or to death from any cause. The second endpoints were overall survival (OS) and distant metastasis-free survival (DMFS). OS is defined as the length of time from the date of surgery on the primary tumor to death from any cause, or to time of last visit. DMFS is defined as the length of time from the date of surgery on the primary tumor to time of distant disease recurrence. Survival 
curves based on PD-L1 expression were estimated using the Kaplan-Meier product-limit method and compared by log-rank test. Univariate Cox proportional hazards models were fit to identify factors significantly related to DFS and OS. To assess whether the expression of PD-L1 by tumor cells was an independent predictor of survival, a multivariate Cox model was constructed to adjust for other patient/clinical characteristics that were significant in the univariate analyses. Two-way interaction terms between PD-L1 expression and other factors in the multivariate Cox model were also assessed. A nomogram was used to predict the positive probability of PD-L1. All analyses were two-sided, and significance was set at a p-value of 0.05. Statistical analyses were performed using SPSS statistics software version 19 and R software 2.15.3.

\section{ACKNOWLEDGMENTS}

This study was supported in part by a grant from the National Natural Science Foundation of China (Grant No. 81272896, 81372502), National High Technology Research and Development Program of China (Grant No.2012AA02A501), Natural Science Foundation of Guangdong (Grant No. S2013010016564) and High Technology Research of Guangdong (Grant No.2012B031800447).

\section{CONFLICTS OF INTEREST}

The authors declare no conflicts of interest.

\section{REFERENCES}

1. Torre LA, Bray F, Siegel RL, Ferlay J, Lortet-Tieulent J and Jemal A. Global cancer statistics, 2012. CA Cancer J Clin. 2015; 65:87-108.

2. Weigelt B, Peterse JL and van 't Veer LJ. Breast cancer metastasis: markers and models. Nat Rev Cancer. 2005; 5:591-602.

3. Davies C, Pan H, Godwin J, Gray R, Arriagada R, Raina V, Abraham M, Medeiros Alencar VH, Badran A, Bonfill X, Bradbury J, Clarke M, Collins R, Davis SR, Delmestri A, Forbes JF, et al. Long-term effects of continuing adjuvant tamoxifen to 10 years versus stopping at 5 years after diagnosis of oestrogen receptor-positive breast cancer: ATLAS, a randomised trial. Lancet. 2013; 381:805-816.

4. Sgroi DC, Sestak I, Cuzick J, Zhang Y, Schnabel CA, Schroeder B, Erlander MG, Dunbier A, Sidhu K, LopezKnowles E, Goss PE and Dowsett M. Prediction of late distant recurrence in patients with oestrogen-receptorpositive breast cancer: a prospective comparison of the breast-cancer index (BCI) assay, 21-gene recurrence score, and IHC4 in the TransATAC study population. Lancet Oncol. 2013; 14:1067-1076.

5. Sgroi DC, Carney E, Zarrella E, Steffel L, Binns SN,
Finkelstein DM, Szymonifka J, Bhan AK, Shepherd LE, Zhang Y, Schnabel CA, Erlander MG, Ingle JN, Porter P, Muss HB, Pritchard KI, et al. Prediction of late disease recurrence and extended adjuvant letrozole benefit by the HOXB13/IL17BR biomarker. J Natl Cancer Inst. 2013; 105:1036-1042.

6. Gettinger S and Herbst RS. B7-H1/PD-1 blockade therapy in non-small cell lung cancer: current status and future direction. Cancer J. 2014; 20:281-289.

7. Thompson RH, Dong H, Lohse CM, Leibovich BC, Blute ML, Cheville JC and Kwon ED. PD-1 is expressed by tumor-infiltrating immune cells and is associated with poor outcome for patients with renal cell carcinoma. Clin Cancer Res. 2007; 13:1757-1761.

8. Chawla A, Philips AV, Alatrash G and Mittendorf E. Immune checkpoints: A therapeutic target in triple negative breast cancer. Oncoimmunology. 2014; 3:e28325.

9. Powles T, Eder JP, Fine GD, Braiteh FS, Loriot Y, Cruz C, Bellmunt J, Burris HA, Petrylak DP, Teng SL, Shen X, Boyd Z, Hegde PS, Chen DS and Vogelzang NJ. MPDL3280A (anti-PD-L1) treatment leads to clinical activity in metastatic bladder cancer. Nature. 2014; 515:558-562.

10. Zielinski CC. A phase I study of MEDI4736, NNT-PD-L1 antibody in patients with advanced solid tumors. Transl Lung Cancer Res. 2014; 3:406-407.

11. Sunshine J and Taube JM. PD-1/PD-L1 inhibitors. Curr Opin Pharmacol. 2015; 23:32-38.

12. Mittendorf EA, Philips AV, Meric-Bernstam F, Qiao N, Wu Y, Harrington S, Su X, Wang Y, Gonzalez-Angulo AM, Akcakanat A, Chawla A, Curran M, Hwu P, Sharma P, Litton JK, Molldrem JJ, et al. PD-L1 expression in triplenegative breast cancer. Cancer Immunol Res. 2014; 2:361370 .

13. Soliman H, Khalil F and Antonia S. PD-L1 expression is increased in a subset of basal type breast cancer cells. PLoS One. 2014; 9:e88557.

14. Muenst S, Schaerli AR, Gao F, Daster S, Trella E, Droeser RA, Muraro MG, Zajac P, Zanetti R, Gillanders WE, Weber WP and Soysal SD. Expression of programmed death ligand 1 (PD-L1) is associated with poor prognosis in human breast cancer. Breast Cancer Res Treat. 2014; 146:15-24.

15. Ghebeh H, Mohammed S, Al-Omair A, Qattan A, Lehe C, Al-Qudaihi G, Elkum N, Alshabanah M, Bin Amer $\mathrm{S}$, Tulbah A, Ajarim D, Al-Tweigeri T and Dermime S. The B7-H1 (PD-L1) T lymphocyte-inhibitory molecule is expressed in breast cancer patients with infiltrating ductal carcinoma: correlation with important high-risk prognostic factors. Neoplasia. 2006; 8:190-198.

16. Gibson J. Anti-PD-L1 for metastatic triple-negative breast cancer. Lancet Oncol. 2015; 16:e264.

17. Schalper KA, Velcheti V, Carvajal D, Wimberly H, Brown J, Pusztai L and Rimm DL. In situ tumor PD-L1 mRNA expression is associated with increased TILs and better 
outcome in breast carcinomas. Clin Cancer Res. 2014; 20:2773-2782.

18. Sabatier R, Finetti P, Mamessier E, Adelaide J, Chaffanet M, Ali HR, Viens P, Caldas C, Birnbaum D and Bertucci F. Prognostic and predictive value of PDL1 expression in breast cancer. Oncotarget. 2015; 6:5449-5464.

19. Gatalica Z, Snyder C, Maney T, Ghazalpour A, Holterman DA, Xiao N, Overberg P, Rose I, Basu GD, Vranic S, Lynch HT, Von Hoff DD and Hamid O. Programmed Cell Death 1 (PD-1) and Its Ligand (PD-L1) in Common Cancers and Their Correlation with Molecular Cancer Type. Cancer Epidemiol Biomarkers Prev. 2014; 23:2965-70.

20. Ali HR, Glont SE, Blows FM, Provenzano E, Dawson SJ, Liu B, Hiller L, Dunn J, Poole CJ, Bowden S, Earl HM, Pharoah PD and Caldas C. PD-L1 protein expression in breast cancer is rare, enriched in basal-like tumours and associated with infiltrating lymphocytes. Ann Oncol. 2015; 26:1488-1493.

21. Azuma K, Ota K, Kawahara A, Hattori S, Iwama E, Harada T, Matsumoto K, Takayama K, Takamori S, Kage M, Hoshino T, Nakanishi Y and Okamoto I. Association of PD-L1 overexpression with activating EGFR mutations in surgically resected nonsmall-cell lung cancer. Ann Oncol. 2014; 25:1935-1940.

22. Akbay EA, Koyama S, Carretero J, Altabef A, Tchaicha JH, Christensen CL, Mikse OR, Cherniack AD, Beauchamp EM, Pugh TJ, Wilkerson MD, Fecci PE, Butaney M, Reibel JB, Soucheray M, Cohoon TJ, et al. Activation of the PD-1 pathway contributes to immune escape in EGFR-driven lung tumors. Cancer Discov. 2013; 3:1355-1363.

23. D'Incecco A, Andreozzi M, Ludovini V, Rossi E, Capodanno A, Landi L, Tibaldi C, Minuti G, Salvini J, Coppi E, Chella A, Fontanini G, Filice ME, Tornillo L, Incensati RM, Sani S, et al. PD-1 and PD-L1 expression in molecularly selected non-small-cell lung cancer patients. $\mathrm{Br}$ J Cancer. 2015; 112:95-102.

24. Schalper KA. PD-L1 expression and tumor-infiltrating lymphocytes: Revisiting the antitumor immune response potential in breast cancer. Oncoimmunology. 2014; 3:e29288. 